\title{
¿FRACASO DEL RECONOCIMIENTO MUTUO DE RESOLUCIONES DE LIBERTAD VIGILADA EN ESPAÑA? UNA REFLEXIÓN SOBRE LA SITUACIÓN DE LOS CONDENADOS EXTRANJEROS DE NACIONALIDAD COMUNITARIA*
}

\section{FAILURE OF THE MUTUAL RECOGNITION OF PROBATION DECISIONS IN SPAIN? REFLECTING ON THE SITUATION OF THE SENTENCED EU NATIONAL FOREIGNERS}

Patricia Faraldo Cabana ${ }^{\star \star}$, Cristina Fernández Bessa ${ }^{\star \star \star}$

Resumen: En este trabajo se analiza el nivel de aplicación del reconocimiento mutuo de resoluciones de libertad vigilada en España, hasta el momento muy escaso pese a que el número de beneficiarios potenciales de la medida (condenados extranjeros de nacionalidad comunitaria) es significativo. Para valorar las razones de esta falta de aplicación, se estudian, en primer lugar, los objetivos tanto de la Decisión Marco 2008/947/JAI como de la Ley 23/2014. A continuación se muestra como la rehabilitación social de los condenados y la protección de las víctimas y del público en general se ponen en relación crítica con la prevalencia de los procedimientos de expulsión de condenados comunitarios. Estas expulsiones siguen una lógica distinta, pensada en clave exclusivamente nacional. Su prevalencia en España ha convertido en innecesario el reconocimiento mutuo, puesto que buena parte de los condenados que podrían beneficiarse de él son expulsados. Surge así un siste-

* Este trabajo ha sido realizado en el marco de la Action Grant Trust and Social Rehabilitation in Action - Trust and Action - Grant Agreement 800829, financiada por el Programa Justicia de la UE 2014-2020 - www.eurehabilitation. unito.it. El contenido recoge las opiniones de los miembros del consorcio de investigación y es de la exclusiva responsabilidad de sus autoras. La Comisión Europea no acepta responsabilidad alguna por el uso que se pueda hacer de la información que contiene.

** Catedrática de Derecho Penal, Universidade da Coruña y adjunct professor, Queensland University of Technology, Australia, patricia.faraldo@udc.es

*** Investigadora posdoctoral, Universidade da Coruña, c.fernandezb@udc.es 
ma incoherente y contradictorio que no tiene en cuenta que el fin constitucional de la privación de libertad es la rehabilitación social del condenado.

Palabras clave: reconocimiento mutuo; libertad vigilada; Decisión Marco 2008/947/JAI; expulsión; condenados extranjeros; ciudadanos comunitarios; rehabilitación social

Abstract: This paper analyses the level of implementation of the mutual recognition of probation decisions in Spain. Despite the significant number of potential beneficiaries of the measure (sentenced EU national foreigners), this mutual recognition instrument has barely been implemented as of 2018. In order to assess the reasons of this lack of implementation, this article begins by examining the objectives and goals of both the Framework Decision 2008/947/JHA and the Spanish Law No. 23/2014. Then, it shows how the social rehabilitation of the convicted individuals and the protection of victims and general public are critically related to the prevalence of deportation procedures of sentenced EU citizens. These deportations are based on a different rationality, in which national interests are given preference, and their prevalence in Spain has rendered the mutual recognition instruments irrelevant since a significant number of the sentenced individuals who stand to benefit from them end up being deported. This analysis lays bare an incoherent and contradictory system which neglects the constitutional purpose of custodial measures, the social rehabilitation of the sentenced individual.

Key words: mutual recognition, probation measures, Framework Decision 2008/947/JHA, deportation, convicted foreigners, EU citizens, social rehabilitation.

\section{INTRODUCCIÓN}

La Ley 23/2014, de 20 de noviembre, de reconocimiento mutuo de resoluciones penales en la Unión Europea ha traspuesto al Derecho interno español, entre otras, la Decisión Marco 2008/947/JAI del Consejo, de 27 de noviembre de 2008, relativa a la aplicación del principio de reconocimiento mutuo de sentencias y resoluciones de libertad vigilada con miras a la vigilancia de las medidas de libertad vigilada y las penas sustitutivas. Esta trasposición tuvo lugar de forma ciertamente atropellada, dadas las prisas derivadas de las san- 
ciones por falta de adaptación de la normativa europea al Derecho interno ${ }^{1}$. Es un ejemplo de las prisas el que no se realizara una verdadera adaptación de la Decisión Marco al Derecho español, limitándose a una cuestionable traducción de sus términos. Baste como ejemplo el uso del término "libertad vigilada" en la Ley 23/2014 para hacer referencia a lo que en la Decisión Marco se denomina en inglés probation decisions. En el Derecho Penal español, la libertad vigilada es una medida de seguridad no privativa de libertad regulada en el art. 106 del Código Penal (en adelante, CP), consistente en el sometimiento del condenado a control judicial a través del cumplimiento por su parte de alguna o algunas de las medidas previstas, que se puede aplicar a inimputables, semiimputables e imputables peligrosos en ciertos $\operatorname{casos}^{2}$. No tiene nada que ver con los mecanismos asimilables a la probation a los que se refiere la Decisión Marco, que en España son la suspensión de la ejecución de las penas privativas de libertad y la libertad condicional ${ }^{3}$.

En cualquier caso, la trasposición de la Decisión Marco 2008/947/ JAI ha permitido aplicar en nuestro país el reconocimiento mutuo de resoluciones relativas a:

- la suspensión de la ejecución de las penas privativas de libertad $(\text { arts. } 80 \text { a } 87 \mathrm{CP})^{4}$;

1 La Decisión Marco debía haberse traspuesto antes del 6 de diciembre de 2011. El retraso respondió a un ánimo codificador por parte del legislador español, que trató de simplificar el disperso panorama normativo que podría existir en caso de que para cada norma de la UE sobre reconocimiento mutuo en materia penal se elaborase una ley distinta. Sin embargo, la implementación de la Decisión Marco 2008/909/JAI provocó ciertos recelos en el Tribunal Supremo, lo que dio lugar a que se retrasara hasta encontrar soluciones interpretativas consideradas aceptables.

2 La figura está perfectamente delimitada tanto en lo que respecta a su naturaleza como a los casos en que se aplica. Vid. por todos Acale Sánchez (2010, pp. 147-158).

3 De hecho, en su Guía sobre el reconocimiento de resoluciones de libertad vigilada, el Servicio de Relaciones Internacionales del Consejo General del Poder Judicial reconoce que "medidas de libertad vigilada... es un concepto jurídico distinto del regulado en el Código Penal” (p. 3) (de acuerdo, Leganés Gómez, 2016).

4 Debe incluirse aquí incluso el caso de que la suspensión se condicione únicamente al pago de la multa impuesta en sustitución de la pena privativa de libertad, pues siempre está condicionada también a que el reo no vuelva a delinquir. En contra, sin embargo, Rodríguez-Medel Nieto y Sebastián Montesinos (2015, p. 341). 
- la libertad condicional (arts. 90 a 92 CP) (Flore et al., 2012, pp. 133-136; Rodríguez-Medel Nieto y Sebastián Montesinos, 2015, p. 341$)$;

- la medida de libertad vigilada (supervised release) para menores (arts. 7.1 h), 15 y 51 de la Ley Orgánica 5/2000, de 12 de enero, reguladora de la responsabilidad penal de los menores, en adelante LORPM) (Flore et al., 2013, pp. 136-138);

- y la pena de trabajos en beneficio de la comunidad (arts. 33, 39, 40, 49 y 53 CP) (Flore et al., 2012, pp. 144-145), pero únicamente cuando es una modalidad de cumplimiento de la responsabilidad personal subsidiaria por impago de multa (art. $53.1 \mathrm{CP}$ ) o una condición de la suspensión de la ejecución de las penas privativas de libertad (art. $84 \mathrm{CP}$ ).

Ahora bien, a pesar de que estamos ante medidas que afectan a un número considerable de condenados extranjeros de nacionalidad comunitaria (1.435 en 20185), lo cierto es que en España apenas se está aplicando su reconocimiento mutuo. Para averiguar los motivos, a continuación se analizarán, en primer lugar, los objetivos que persiguen tanto la Decisión Marco 2008/947/JAI como la Ley 23/2014; en segundo lugar, se expondrá el nivel de aplicación que el reconocimiento mutuo de resoluciones de libertad vigilada ha tenido hasta el momento en España, poniéndolo en relación con el número de beneficiarios potenciales (condenados comunitarios); en tercer lugar, se avanzará una primera hipótesis explicativa de los resultados encontrados, que tiene que ver con la prevalencia de los procedimientos de expulsión de condenados comunitarios, mejor conocidos y con una trayectoria más larga de uso que el de reconocimiento mutuo; en cuarto lugar, se pondrán de manifiesto las incoherencias y contradicciones en que incurre el sistema español; por último, se terminará con unas conclusiones.

\section{LOS OBJETIVOS DEL RECONOCIMIENTO MUTUO DE RESOLUCIONES DE LIBERTAD VIGILADA}

La Decisión Marco 2008/947/JAI ha venido a completar el Programa de medidas destinadas a poner en práctica el principio de

5 Cifra de penados a penas y medidas alternativas a la prisión en stock en 2018, según datos del Ministerio del Interior (2019, pp. 345-347). 
reconocimiento mutuo de las resoluciones judiciales en materia penal, aprobado por el Consejo el 30 de noviembre de 2000 (DO C 12 de 15.1.2001). El objetivo, entonces y ahora, era "garantizar la cooperación cuando se trate de una persona sometida a obligaciones o medidas de vigilancia y de asistencia con arreglo, en particular, a una condena en suspenso o a la libertad condicional". En él se insistió tanto en el Libro Verde sobre la aproximación, el reconocimiento mutuo y la ejecución de penas en la Unión Europea (COM/2004/0334 final), y en el Programa de La Haya: consolidación de la libertad, la seguridad y la justicia en la Unión Europea (DO C 53 de 3.3.2005), como en el Plan de Acción del Consejo y la Comisión por el que se aplica el Programa de La Haya sobre refuerzo de la libertad, la seguridad y la justicia en la Unión Europea (DO C 198 de 12.8.2005), que incluía en su lista de medidas para reforzar la cooperación judicial en asuntos penales una "Propuesta referente al reconocimiento y ejecución de las sanciones alternativas y a las condenas condicionales", a presentar en 2007. En este contexto, a iniciativa de la República Federal de Alemania y de la República Francesa, se presentó en 2007 la Propuesta de una Decisión Marco "relativa al reconocimiento y vigilancia de penas suspendidas, penas sustitutivas y condenas condicionales" (DO C 147/1 de 30.6.2007). Su adopción supuso el reconocimiento de la importancia de estas medidas tanto para el objetivo de crear un espacio de libertad, seguridad y justicia, como para el de conseguir la rehabilitación y reinserción de los condenados extranjeros (van Kalmthout y Durnescu, 2008, p. 41; Cid Moliné, 2010, pp. 268-270). Pero no solo eso. El considerando $8^{\circ}$ de la Decisión Marco explica que los objetivos que se persiguen con su adopción son, por un lado, incrementar las posibilidades de reinserción social del condenado al permitirle mantener sus lazos familiares, lingüísticos, culturales y de otra índole, y, por otro, mejorar el control del cumplimiento de las medidas de libertad vigilada y de las penas sustitutivas con objeto de evitar la reincidencia y de este modo tener en cuenta el principio de la protección de las víctimas y del público en general. El primer inciso del art. 1, dedicado a los "Objetivos y ámbito de aplicación", insiste en las mismas ideas.

La Ley 23/2014 sigue una orientación similar. En el apartado VIII del Preámbulo se indica que su objetivo es incrementar las posibilidades de reinserción social del condenado al permitirle mantener sus lazos familiares, lingüísticos y culturales, así como mejorar el control del cumplimiento de las medidas de libertad 
vigilada y de las penas sustitutivas con objeto de evitar la reincidencia, teniendo en cuenta el principio de la protección de las víctimas.

La preocupación por la reinserción social de los condenados extranjeros está fundada. Aunque en los sistemas penal y penitenciario de los Estados miembros de la Unión Europea rige el principio de no discriminación, en la práctica los extranjeros se ven privados en muchas ocasiones de la aplicación de alternativas a la prisión o formas de liberación anticipada simplemente por esa condición de no nacionales (van Kalmthout et al., 2007; Bhui, 2009, pp. 161-162; Knapen, 2010, p. 118; Banks, 2011; Kaufman, 2012; McNally y Burke, 2012, pp. 70-71; Ugelvik, 2014, pp. 113, 116117; Warr, 2016; Durnescu, 2017, p. 357). Los extranjeros reciben más penas de prisión que los nacionales, sin poder acceder a la suspensión de la ejecución o a penas no privativas de libertad ante el temor de los jueces de que se fuguen, y que cuando ingresan en prisión se quedan más tiempo en ella, sin poder acceder a la libertad condicional, sin olvidar que cuando son puestos en libertad en muchos casos es para ser expulsados inmediatamente. Por tanto, la existencia de un mecanismo destinado a permitir que estas personas cumplan la libertad condicional, el período de suspensión de la ejecución de la pena privativa de libertad o las penas sustitutivas en su país de origen o de residencia no puede sino aplaudirse. Ahora, bien, ¿`se está haciendo uso de ese instrumento?

\section{2. ¿SE UTILIZA EL RECONOCIMIENTO MUTUO DE RESOLUCIONES DE LIBERTAD VIGILADA EN ESPAÑA?}

El número de beneficiarios potenciales del reconocimiento mutuo de resoluciones de libertad vigilada en nuestro país es elevado. A continuación, se ofrecen las cifras oficiales en la materia.

Según el Instituto Nacional de Estadística (INE) condenas de personas adultas en España en 2018 (último año del que se tienen cifras) se impusieron a extranjeros, alcanzándose la cifra de 67.942 de un total de 286.637 personas adultas condenadas por

6 Disponibles en https://www.ine.es/prensa/ec_am_2018.pdf, y en http:// www.poderjudicial.es/cgpj/es/Temas/Estadistica-Judicial/Estadistica-por-temas/ Datos-penales--civiles-y-laborales/Delitos-y-condenas/Condenados--explotacionestadistica-del-Registro-Central-de-Penados-/ 
sentencia firme registradas en el Registro Central de Penados. Casi la tercera parte del total de extranjeros, el 32,7\%, pertenecía a países comunitarios (sin contar España): 22.218 personas. En relación con los menores de 14 a 17 años, los porcentajes son más reducidos: de un total de 13.664 menores condenados, 2.502, el 18,3\%, son extranjeros. De ellos, 568, el 21,4\% del total de menores extranjeros condenados, son ciudadanos comunitarios (sin contar España).

Si analizamos la situación en las prisiones españolas en el año de referencia, 2018, resulta que los extranjeros constituían el 28,1\% de la población penitenciaria, esto es, había 16.536 internos extranjeros a 31 de diciembre de 2018 (Ministerio del Interior, 2019, p. 308). Ese año, 3.910 internos tenían nacionalidad comunitaria (esta cifra incluye internos en prisión preventiva).

Recapitulando, en 2018 los extranjeros constituían casi el 10\% de la población residente en España, el 23,7\% de los condenados y el $28,1 \%$ de la población penitenciaria. La sobrerrepresentación de los extranjeros en las estadísticas criminales y penitenciarias es evidente. La tasa de condenados extranjeros por cada 1.000 habitantes fue de 17,7, casi el triple que la de los españoles, 6,3. En menores, las cifras son similares. La tasa de condenados extranjeros menores por cada 1.000 habitantes es de 16,3 de nuevo casi triplicando la de menores españoles, 6,5.

La sobrerrepresentación de los extranjeros también tiene que ver con las condenas que reciben. Según el INE, en 2018, por nacionalidad del infractor adulto, las penas privativas de libertad fueron más frecuentes entre los condenados extranjeros (20,7\% del total, frente al 19,3\% de los españoles). En particular, la pena de prisión supuso el $20,3 \%$ del total en los extranjeros y el $18,5 \%$ en los españoles. Los comunitarios reciben penas privativas de libertad en el 19\% de los casos, ligeramente menos que los españoles, pero penas de prisión en un porcentaje ligeramente superior a estos, el 18,6\%. Además, acceden a la libertad condicional en un porcentaje inferior al de los españoles. En el caso de menores, el internamiento en régimen cerrado también se aplica más a extranjeros que a españoles $(4,3 \%$ frente al 1,3\%). Por su parte, las medidas de internamiento en régimen semiabierto y de libertad vigilada recayeron con más frecuencia en extranjeros $(15,7 \%$ y $45,1 \%$ del total, respectivamente) que en españoles (9,5\% y 42,3\%). En cambio, los extranjeros recibieron con menor frecuencia las medidas de realización de tareas socioeducativas y prestación en beneficio de la comunidad (8,7\% y 13,5\% 
del total, respectivamente, frente a $12,9 \%$ y $15,7 \%$ de los españoles) $)^{7}$.

No es objeto de este trabajo analizar las razones de esta situación, abundantemente exploradas en la doctrina española (García España, 2007, p. 108; Monclús Masó, 2006, p. 192; García España et al., 2012, p. 101; Fernández Bessa y Brandariz García, 2016; González Sánchez, 2016, pp. 134 ss.) e internacional (Delgrande y Aebi, 2010, p. 143; Aebi, 2016, pp. 136-137), pero sí subrayar que España es un claro ejemplo de la discriminación que experimentan los ciudadanos extranjeros en el sistema penal. Esa situación discriminatoria se puede observar tanto en el momento de imposición de la sanción como a la hora de adoptar medidas que evitan el ingreso en prisión o acortan la estancia, como la suspensión de la ejecución y la libertad condicional, según viene denunciando reiteradamente la doctrina especializada desde hace años (Sánchez Yllera, 1990, pp. 65-70; Leganés Gómez, 2002; Rodríguez Yagüe, 2004; Ruiz Rodríguez, 2006, pp. 181-193; García España, 2007, pp. 101-134; Brandariz García, 2010, pp. 103-132; de Marcos Madruga, 2010; Blanco Cordero y Doval Pais, 2013). Ahora bien, pese a la reconocida situación de discriminación de los extranjeros en el sistema penal y penitenciario y al elevado número de condenados de nacionalidad comunitaria, hasta el momento ha habido pocas resoluciones de reconocimiento mutuo de estas medidas, sea emitidas que recibidas.

TABLA 1A

SOLICITUDES DE RECONOCIMIENTO DE RESOLUCIÓN POR LA QUE SE IMPONE UNA MEDIDA DE LIBERTAD VIGILADA (EMITIDAS)

\begin{tabular}{|l|c|c|c|c|}
\hline Órgano judicial & 2015 & 2016 & 2017 & 2018 \\
\hline Audiencia Nacional & 0 & 0 & 0 & 0 \\
\hline Audiencias provinciales & 0 & 0 & 2 & 2 \\
\hline Juzgados Centrales de Instrucción & 0 & 0 & 0 & 0 \\
\hline Juzgados de Violencia contra la Mujer & 0 & 0 & 0 & 0 \\
\hline Juzgados de 1a instancia, Instrucción y 1ª Instancia e Instrucción & 0 & 0 & 0 & 0 \\
\hline Juzgados de lo Penal & 0 & 6 & 2 & 1 \\
\hline TOTAL & 0 & 6 & 4 & 3 \\
\hline
\end{tabular}

7 A diferencia de las estadísticas de condenados adultos, en el caso de menores no se ofrecen datos desagregados dentro de la categoría de extranjeros, por lo que no se pueden dar cifras sobre comunitarios. 
TABLA 1B

SOLICITUDES DE RECONOCIMIENTO DE RESOLUCIÓN POR LA QUE SE IMPONE UNA MEDIDA DE LIBERTAD VIGILADA (RECIBIDAS)

\begin{tabular}{|l|c|c|c|c|}
\hline Órgano judicial & 2015 & 2016 & 2017 & 2018 \\
\hline Juzgado Central de lo Penal & 0 & 3 & 5 & nd \\
\hline Juzgado Central de Menores & 0 & 0 & 0 & nd \\
\hline TOTAL & 0 & 3 & 5 & nd \\
\hline
\end{tabular}

Fuente: Consejo General del Poder Judicial ${ }^{8}$ y comunicación personal con el jefe de sección del Servicio de Relaciones Internacionales del Consejo.

La situación no parece muy distinta a nivel internacional. En general, la doctrina reconoce el bajo nivel de aplicación que se ha alcanzado hasta el momento, aun lamentando la falta de datos fiables (Durnescu, 2017, pp. 361-362). ¿A qué se debe este escaso uso?

\section{UNA PRIMERA HIPÓTESIS EXPLICATIVA DE LA ESCASA APLICACIÓN DEL RECONOCIMIENTO MUTUO: LA PREVALENCIA DE LOS PROCEDIMIENTOS DE EXPULSIÓN DE CONDENADOS COMUNITARIOS ${ }^{9}$}

Las razones de la escasa aplicación del procedimiento de reconocimiento mutuo de resoluciones de libertad vigilada no han sido estudiadas hasta el momento. En nuestra opinión, tienen mucho que ver con la prevalencia, en términos cuantitativos, de los procedimientos de expulsión de condenados comunitarios. Esa prevalencia se relaciona directamente con dos factores. El primero, el interés

8 Disponibles en http://www.poderjudicial.es/cgpj/es/Temas/EstadisticaJudicial/Estadistica-por-temas/Aspectos-internacionales/Cooperacion-conorganos-judiciales-extranjeros/Solicitudes-de-cooperacion-tramitadasdirectamente-por-los-organos-judiciales/

$9 \quad$ No son objeto de análisis en este apartado otras medidas previstas para facilitar la expulsión de extranjeros que han cometido un delito, pero antes de la condena, como el archivo de la causa penal contra el extranjero con el objeto de "favorecer la realización de los fines de la política inmigratoria" (art. 2 bis de la Ley Orgánica 4/2000, de 11 de enero, sobre derechos y libertades de los extranjeros en España y su integración social, en adelante LOEx) eliminando los obstáculos para su expulsión administrativa, recogido en el art. 57.7 LOEx. 
del Estado en hacerlos funcionar, del que son prueba las declaraciones del Ministerio del Interior en el pasado reciente según las cuales una prioridad capital de la política migratoria española es el internamiento de "extranjeros delincuentes" y la ejecución de las expulsiones vinculadas con la criminalidad (Boza Martínez, 2016, pp. 117 ss.; Brandariz García, 2016, pp. 190-191). El segundo factor es el mayor desarrollo práctico de los procedimientos de expulsión de condenados extranjeros, viejos conocidos del Ordenamiento jurídico español, frente a la novedad del reconocimiento mutuo, lo que hace que cuando ambas opciones estén abiertas se prefiera la expulsión, incluso cuando están implicados comunitarios. A continuación, se describen sucintamente tanto la sustitución de las penas de prisión por la expulsión prevista en el Código Penal, como la expulsión administrativa por razones de orden público o seguridad pública que recoge para comunitarios el Real Decreto 240/2007, de 16 de febrero, sobre entrada, libre circulación y residencia en España de ciudadanos de los Estados miembros de la Unión Europea y de otros Estados parte en el Acuerdo sobre el Espacio Económico Europeo. El objetivo no es realizar un estudio en profundidad, imposible por razones de espacio, sino exponer las líneas básicas de las respectivas normativas.

$\mathrm{El}$ art. 89.1 CP prevé la sustitución de las penas de prisión de más de un año impuestas a un ciudadano extranjero por su expulsión del territorio español. En el caso de que se imponga una pena de más de cinco años de prisión, o varias penas que excedan de esa duración, se cumplirán en España, pero solo "en la medida en que resulte necesario para asegurar la defensa del orden jurídico y restablecer la confianza en la vigencia de la norma infringida por el delito" (art. 89.2 CP). "No procederá la sustitución cuando, a la vista de las circunstancias del hecho y las personales del autor, en particular su arraigo en España, la expulsión resulte desproporcionada" (art. 89.4 CP) ${ }^{10}$. En el caso

10 El art. 89 CP obliga a un juicio de proporcionalidad. El arraigo del extranjero es, por tanto, un límite frente a la expulsión no solo en el momento de dictar sentencia, sino también en el momento de su ejecución (Roig Torres, 2014; García España, 2018). De esta manera, la consideración de la expulsión como desproporcionada a la luz del arraigo supondría una limitación absoluta a su ejecución (conclusión $7^{\mathrm{a}}$ de la Circular 7/2015 de la Fiscalía General del Estado, sobre la expulsión de ciudadanos extranjeros como medida sustitutiva de la pena de prisión tras la reforma operada por LO 1/2015). 
de comunitarios ${ }^{11}$, la expulsión se rodea de mayores cautelas, pues solamente procederá cuando represente una amenaza grave para el orden público o la seguridad pública en atención a la naturaleza, circunstancias y gravedad del delito cometido, sus antecedentes y circunstancias personales (art. 89.4 CP). Incluso así, en 2015 se impuso la expulsión penal a 58 ciudadanos comunitarios, a 63 en 2016, a 53 en 2017 y a 83 en $2018^{12}$.

La sustitución de la pena de prisión por expulsión para condenados extranjeros se puede considerar un sub-sistema dentro del régimen de control migratorio en España, que es sustancialmente de Derecho administrativo (Brandariz García y Fernández Bessa, 2017, pp. 329-330). Aunque este sub-sistema penal se había caracterizado tradicionalmente por un nivel de ejecución deficiente (García España, 2007, pp. 120-121; Brandariz García, 2011, pp. 85 ss.), habiendo sido considerado como un componente subordinado del régimen de control migratorio (Monclús Masó, 2008, pp. 492 ss.; Daunis Ro-

11 Estas cautelas no son aplicables a ciudadanos de los países miembros del Acuerdo del Espacio Económico Europeo (Islandia, Liechtenstein, Noruega y Suiza). Solo se asimilan a los comunitarios en materia de expulsiones administrativas.

12 Según la explotación estadística del Consejo General del Poder Judicial de los datos del Registro Central de Penados, estadística condenados adultos — penas, disponible en la página web http:/www.poderjudicial.es/cgpj/es/Temas/ Estadistica-Judicial/Estadistica-por-temas/Datos-penales--civiles-y-laborales/ Delitos-y-condenas/Condenados--explotacion-estadistica-del-Registro-Centralde-Penados- /. Ahí consta que la expulsión penal se impuso a 39 ciudadanos comunitarios en 2014, 32 en 2013 y 31 en 2012. En 2011 no hay datos. Esto es llamativo, porque antes de la reforma de 2015 el art. 89 CP solo era aplicable a ciudadanos extranjeros sin residencia legal en España, concepto en el que cabe entender que no entran los comunitarios, sobre la base de su derecho "a entrar, salir, circular y residir libremente en territorio español” (art. 3 RD 240/2007 y normativa comunitaria concordante). En este sentido apuntaba un sector doctrinal (Úbeda de los Cobos, 2006, p. 1442; Fernández Arévalo, 2010; Brandariz García, 2011, pp. 173-176). No obstante, otro sector admitía la expulsión penal de comunitarios, sobre la base de que una cosa es el derecho de residir y otra la condición jurídica de residente (Cugat Mauri, 2001, p. 33; Aranda Carbonel, 2007, p. 291, con puntualizaciones). Los datos indican que la expulsión penal también se imponía a ciudadanos comunitarios. La Fiscalía General del Estado (2014, p. 389) limitaba esta posibilidad a "la existencia previa de resolución administrativa de expulsión dictada en aplicación del RD 240/2007 de 16 de febrero, sobre entrada, libre circulación y residencia en España de ciudadanos de los Estados Miembros de la Unión Europea y de otros Estados Parte en el Acuerdo sobre el Espacio Económico Europeo". 
dríguez, 2009), esta situación cambió sustancialmente entre 2008 y 2012 (Fernández Bessa, 2013, pp. 68 ss.). En efecto, el número de expulsiones penales ejecutadas de condenados extranjeros pasó de 634 en 2008 a 2.309 en 2012. Desde entonces su número se ha ido reduciendo, siguiendo la tendencia general de disminución del número de expulsiones. En 2017 se ejecutaron 988 expulsiones por este motivo y 962 en $2018^{13}$. No olvidemos que en estas cifras de expulsiones penales se incluyen los condenados comunitarios, aunque originalmente no fueran ellos los objetivos prioritarios de la política migratoria oficial.

En cuanto a la expulsión administrativa, a los ciudadanos de la Unión Europea y de los Estados miembros del Acuerdo del Espacio Económico Europeo, así como a sus parejas y ciertos parientes no se les aplica la Ley Orgánica 4/2000, sino el Real Decreto 240/2007, de 16 de febrero, ya citado. Su art. 15.1 permite ordenar la expulsión o devolución de un ciudadano comunitario o asimilado "cuando así lo impongan razones de orden público, de seguridad pública o de salud pública", puntualizando en caso de ser residentes que solo podrán ser expulsados "si existen motivos graves de orden público o seguridad pública. Asimismo, antes de adoptarse una decisión en ese sentido, se tendrán en cuenta la duración de la residencia e integración social y cultural del interesado en España, su edad, estado de salud, situación familiar y económica, y la importancia de los vínculos con su país de origen". El art. 15.5 d) advierte que "cuando [la decisión de expulsión] se adopte por razones de orden público o de seguridad pública, deberán estar fundadas exclusivamente en la conducta personal de quien sea objeto de aquéllas, que, en todo caso, deberá constituir una amenaza real, actual y suficientemente grave que afecte a un interés fundamental de la sociedad, y que será valorada, por el órgano competente para resolver, en base a los informes de las Autoridades policiales, fiscales o judiciales que obren en el expediente. La existencia de condenas penales anteriores no constituirá, por sí sola, razón para adoptar dichas medidas".

Pese a estas cautelas, las expulsiones administrativas de comunitarios y asimilados han aumentado en los últimos años. También su proporción respecto de las expulsiones ejecutadas ha

13 Sobre estos datos, vid. Boza Martínez (2016, p. 117), Fernández Bessa y Brandariz García (2016, p. 11) y Brandariz García y Fernández Bessa (2017, p. 133). 
crecido en el mismo período, pese a la disminución en números totales: constituyeron el 0,2\% del total en 2008, pero en 2016 llegaron al 7,4\% (Brandariz García y Fernández Bessa, 2017, p. 133). En esas cifras se incluyen las expulsiones por razones de salud pública, orden público o seguridad pública (art. 15 RD 240/2007), que solo se desagregan a partir de 2014. Como se puede ver en la Tabla 2, en 2015 fueron expulsados 103 ciudadanos comunitarios por razones de orden público o seguridad pública, cifra que subió a 179 en 2018.

\section{TABLA 2}

CUADRO ESTADÍSTICO DE EXPULSIONES PENALES Y ADMINISTRATIVAS POR RAZONES DE ORDEN PÚBLICO O SEGURIDAD PÚBLICA DE CIUDADANOS EXTRANJEROS Y COMUNITARIOS EJECUTADAS (2008-2018)

\begin{tabular}{|l|c|c|c|c|c|c|c|c|c|c|c|}
\hline Concepto & 2008 & 2009 & 2010 & 2011 & 2012 & 2013 & 2014 & 2015 & 2016 & 2017 & 2018 \\
\hline $\begin{array}{l}\text { Expulsiones } \\
\text { penales de } \\
\text { extranjeros (art. } \\
\text { 89 CP) } \\
\text { (de los cuales, eran } \\
\text { comunitarios) }\end{array}$ & 634 & 1446 & 1799 & 2301 & 2309 & 2105 & 1916 & 1951 & 1348 & 988 & 962 \\
\hline $\begin{array}{l}\text { Expulsiones } \\
\text { administrativas de } \\
\text { comunitarios } \\
\text { (de las cuales, por } \\
\text { infracciones de } \\
\text { orden público cod. } \\
\text { pen. 0 adm. 0 de } \\
\text { seguridad pública) } \\
\text { (de las cuales, } \\
\text { por razones de } \\
\text { orden público } \\
\text { o seguridad } \\
\text { pública, art. 15 RD } \\
\text { 240/2007) }\end{array}$ & 21 & 11 & 27 & 57 & 166 & 208 & 269 & 398 & 374 & $340^{\star \star \star *}$ & 298 \\
\hline
\end{tabular}

Fuente: Ministerio del Interior, tras sendas solicitudes tramitadas en abril de 2017 y enero de 2019 a través del Portal de Transparencia, y Defensor del Pueblo (2018) y (2019).

*Esta categoría se desagrega desde el 1 de julio de 2015.

**Esta categoría solo aparece desagregada de las razones de salud pública a partir de 2014.

***Esta cifra se ha obtenido restando el número de comunitarios expulsados penalmente al total de ciudadanos con nacionalidad de alguno de los estados miembros de la UE materializadas en 2018 (Defensor del Pueblo, 2019, Anexo 2, p. 8-9). 
En conjunto, pues, las expulsiones de condenados comunitarios se incrementaron entre 2008 y 2017. Sin duda, estas expulsiones alivian al menos en parte la presión que, de otra forma, se dirigiría al reconocimiento mutuo. Además, es razonable pensar que su aplicación es más sencilla para las autoridades españolas, que conocen bien el procedimiento y tienen establecidos ya protocolos de actuación, mientras que el reconocimiento mutuo de medidas de libertad vigilada sigue siendo relativamente desconocido tanto para los operadores jurídicos como para los potenciales beneficiarios.

\section{4. ¿UN SISTEMA COHERENTE? SUPERPOSICIONES Y CONTRADICCIONES}

Una vez que se pone en relación la normativa española relativa a los condenados comunitarios con el reconocimiento mutuo de las medidas de libertad vigilada y el traslado de presos, cabe apreciar que se producen superposiciones y aparecen ciertas contradicciones. Por razones de espacio, nos ceñiremos a las más llamativas.

Hay que resaltar, en primer lugar, que la condena de un ciudadano comunitario a pena de prisión superior a un año puede dar lugar, por una parte, a la expulsión administrativa por razones de orden público o de seguridad pública (art. 15 RD 240/2007), a la sustitución de toda la pena de prisión impuesta o de una parte de ella por la expulsión penal, que se ordenará en todo caso en cuanto obtenga el tercer grado o la libertad condicional (art. 89.1 CP), por otra, y al reconocimiento mutuo de la pena privativa de libertad con traslado del preso a un centro penitenciario en su país de residencia (arts. 63 y siguientes de la Ley 23/2014), por último. Obviamente, las posibilidades de decisión del condenado y las consecuencias para él son distintas. Tanto la expulsión administrativa como la penal y el traslado del preso se pueden realizar sin consentimiento de la persona afectada. Que la expulsión administrativa tenga lugar sin necesidad de consentimiento del afectado es lógico, dada su finalidad. Que así ocurra en el caso de la expulsión penal y del traslado vía reconocimiento mutuo suscita, como poco, cierta sorpresa. En el primer caso, porque las penas privativas de libertad deben orientarse a la rehabilitación y reinserción social del penado, siendo dudoso que su sustitución en todo o en parte por la expulsión en contra de la volun- 
tad del condenado atienda a esa orientación, sin olvidar que en cualquier caso reduce el efecto preventivo de la pena (Guisasola Lerma, 2010; Brandariz García, 2011; Rodríguez Yagüe, 2012; García España, 2016). En el segundo caso, de manera similar, porque, siendo el objetivo declarado del traslado de presos la rehabilitación social, es dudoso que pueda conseguirse trasladando al condenado a un país en el que no quiere estar (de Wree et al. 2009, p. 118; Knapen, 2010, p. 123; Vermeulen et al., 2011, p. 15; de Bondt y Suominen, 2015, pp. 348-349; Martufi, 2018). Las consecuencias para el condenado son distintas: tratándose de expulsión administrativa o penal, no cumple la totalidad o parte de la pena de prisión impuesta, regresa a su país de residencia, donde no recibe tratamiento ni asistencia, y solo está sometido a la prohibición de regresar a España; tratándose de traslado, regresa a su país de residencia a cumplir la pena de prisión, con ingreso en un centro penitenciario.

También la libertad condicional puede dar lugar a distintas posibilidades de intervención y consecuencias para el condenado: expulsión penal (art. 89.2 CP), reconocimiento mutuo para su cumplimiento en el país de residencia (art. 93.1 a) de la Ley 23/2014) o cumplimiento en España. La autorización para el cumplimiento de la libertad condicional en el país de residencia (art. 197 RP), por su parte, solo se aplica, en principio, a "extranjeros no residentes legalmente en España", situación que en el caso de comunitarios se limita a los que tienen orden de expulsión administrativa vía Real Decreto 240/2007, y a "españoles residentes en el extranjero". En la doctrina se acepta, no obstante, que se aplique a comunitarios que cumplen condena de prisión en España, sobre la base del principio de no discriminación con los ciudadanos españoles (Baras González, 2012, p. 69; Montero Pérez de Tudela, 2015, pp. 8-9). La expulsión se puede realizar sin el consentimiento de la persona afectada, a la que simplemente se le da audiencia, mientras que la autorización para el cumplimiento de la libertad condicional en el país de residencia solo se concede "previa conformidad documentada del interno". Por su parte, el reconocimiento mutuo de la medida de libertad vigilada presupone el consentimiento del condenado, pues uno de los requisitos para emitir la resolución es "que [el condenado] haya regresado al Estado donde reside legal y habitualmente o que, aun estando en nuestro país, haya manifestado su voluntad de regresar a éste o a otro Estado miembro que lo autorice" (art. 96.1 c) de la Ley 23/2014). Para comprobar ese consentimiento 
cuando está en España, “con carácter previo a la transmisión de la resolución de libertad vigilada, la autoridad judicial preguntará a la persona condenada si desea regresar o permanecer en su Estado de residencia, concediéndole a tal efecto un plazo de treinta días" (art. 98.2, primer inciso, de la Ley 23/2014). En ninguna parte se exige que esa manifestación de voluntad sea fruto de un consentimiento libre e informado, adoptado con asistencia letrada y, de ser necesario, intérprete, ni tampoco se determina qué hacer si el condenado cambia de opinión y la revoca. Se trata, pues, de un consentimiento que no está rodeado de suficientes garantías (European Agency for Fundamental Rights, 2016, pp. 30 y 98; Durnescu, 2017, p. 362). La expulsión, además, sustituye obligatoriamente a la libertad condicional, de forma que el condenado no tiene que cumplir ninguna condición más allá de la prohibición de regresar a España. Supone la renuncia al cumplimiento de la parte restante de la pena. En este caso, la ejecución de la pena no puede tender a la resocialización, dado que está abocada desde el principio a la expulsión (Ruiz Rodríguez, 2009, pp. 199 y 208; Guisasola Lerma, 2010, p. 135; Rodríguez Yagüe, 2012, p. 15; Blanco Cordero y Doval Pais, 2013, p. 55; González Sánchez, 2015, p. 274). En el caso de la autorización para el cumplimiento de la libertad condicional en el país de residencia prevista en el Reglamento Penitenciario, el Juez de Vigilancia Penitenciaria puede adoptar "cautelas..., en su caso, al objeto de que dicha libertad se disfrute efectivamente en el país fijado" (art. 197.1 RP). Al no tratarse de una expulsión, la salida del territorio español no se acompaña de una prohibición de regreso a España. En el caso del reconocimiento mutuo, siendo España Estado de emisión, la resolución transmite al Estado de ejecución la competencia tanto para la vigilancia de las medidas de libertad vigilada, como para adoptar resoluciones ulteriores en relación con ella, salvo cuando el Estado de ejecución haya hecho declaración en contrario (art. 99 de la Ley 23/2014). Además, también son objeto de transmisión "las medidas que, en su caso, se hubieren impuesto al condenado para el cumplimiento de la responsabilidad civil derivada del delito y demás responsabilidades pecuniarias, cuya satisfacción se debe acreditar por el mismo" (art. 98.4 de la Ley 23/2014).

En los casos aquí reseñados, pues, tanto las posibilidades de decisión del condenado, como las consecuencias de las diversas opciones para él son, como se ha podido comprobar, bien distintas, sin que se establezca criterio alguno que permita una priorización entre ellas. 
Los datos indican que lo que predomina es el cumplimiento en España, seguido por la expulsión penal y la autorización para el cumplimiento en el país de residencia. El reconocimiento mutuo, sea de penas de prisión o de medidas de libertad vigilada, se utiliza muy poco.

\section{TABLA 3}

CUADRO ESTADÍSTICO DE EXCARCELACIONES DE INTERNOS EXTRANJEROS (2012-2017)*

\begin{tabular}{|c|c|c|c|c|c|c|}
\hline Concepto & 2012 & 2013 & 2014 & 2015 & 2016 & 2017 \\
\hline $\begin{array}{l}\text { Expulsión penal sustitutiva de una pena de } \\
\text { prisión inferior a } 6 \text { años (art. } 89.1 \mathrm{CP} \text { ) }\end{array}$ & 671 & 579 & 456 & 418 & 262 & 291 \\
\hline $\begin{array}{l}\text { Expulsión penal al cumplir parte de la pena } \\
0 \text { acceder al tercer grado o a la libertad } \\
\text { condicional (art. } 89.1 \text { y } 2 \mathrm{CP} \text { ) }\end{array}$ & 143 & 225 & 213 & 263 & 391 & 387 \\
\hline $\begin{array}{l}\text { Traslado a país de residencia para cumplimiento } \\
\text { de condena de prisión (Convenio de } \\
\text { Estrasburgo, tratados bilaterales, Ley 23/2014) } \\
\text { (de los cuales, traslados efectuados bajo la } \\
\text { cobertura de la DM 2008/909/JAl y la Ley } \\
\text { 23/2014) }\end{array}$ & 226 & 186 & 163 & $\begin{array}{l}152 \\
(137)\end{array}$ & $\begin{array}{l}109 \\
(96)\end{array}$ & $\begin{array}{l}113 \\
(100)\end{array}$ \\
\hline $\begin{array}{l}\text { Autorización para el cumplimiento de la libertad } \\
\text { condicional en el país de residencia (art. } 197 \text { RP) }\end{array}$ & 626 & 509 & 470 & 414 & 322 & 212 \\
\hline $\begin{array}{l}\text { Cumplimiento de la libertad condicional en } \\
\text { España }\end{array}$ & 1473 & 1347 & 1229 & 1216 & 981 & 892 \\
\hline
\end{tabular}

Fuente: Informe General 2017 de Instituciones Penitenciarias.

* No se ofrecen datos de 2018 porque en el momento de publicación de este artículo (28 de octubre de 2019) todavía no se ha publicado el Informe General 2018 de Instituciones Penitenciarias.

** Los traslados de presos bajo la cobertura de la Ley 23/2014 empezaron en 2015. Estos datos corresponden a la Administración General del Estado.

En el caso de comunitarios, además, resulta llamativo que la expulsión penal solo proceda cuando el condenado represente una amenaza grave para el orden público o la seguridad pública (art. 89.4 CP). Esto significa que si el ciudadano comunitario representa una amenaza grave puede ser expulsado sin más, esto es, sin verse sometido a ninguna medida de tratamiento, asistencia o control, así como tampoco a ninguna prohibición salvo la de no regresar a España, mientras que si no es peligroso cumplirá al menos parte de la pena en España, y al alcanzar el tercer grado o la libertad condicional podrá ser autorizado a cumplir el resto de su condena en su país de residencia, sometido a cautelas cuya naturaleza no se precisa, o bien España 
puede iniciar el procedimiento de reconocimiento mutuo para que cumpla en su país de residencia, sometido a las medidas que se establezcan. Resulta evidente que esta situación, debida a que algunas normas se elaboran desde una perspectiva exclusivamente nacional, mientras que otras siguen una perspectiva europea, no es razonable.

\section{CONCLUSIONES}

En la doctrina europea se ha venido poniendo de manifiesto desde hace años que los mecanismos de reconocimiento mutuo para el traslado de presos y resoluciones de libertad vigilada se han diseñado más en clave de protección del interés de los Estados de emisión en vaciar de extranjeros sus sobrecargados sistemas penitenciarios que de aplicar efectivamente el principio de reinserción y rehabilitación social a los condenados extranjeros (Vermeulen, 2007, p. 353; de Wree et al., 2009, p. 117; Morgenstern, 2009, p. 138; Knapen, 2010, pp. $122-$ 123; Mitsilegas, 2016, p. 222; Neveu, 2016, p. 440). Sin embargo, en España ese temor a la utilización del reconocimiento mutuo en clave migratoria no se ha materializado. El motivo es que ya funcionan otros instrumentos nacionales para conseguir ese mismo efecto, instrumentos que tienen una incidencia directa sobre los condenados comunitarios, pese a las cautelas establecidas para este supuesto.

Esta situación no es deseable, por varias razones. En primer lugar, la expulsión penal es muy criticada por su incapacidad para cumplir los fines de prevención general y especial de la pena y ser contraria a la individualización de la pena y a la necesaria orientación de las penas privativas de libertad a la rehabilitación y reinserción social de los condenados. Se está aplicando precisamente a los condenados que más se podrían beneficiar de la aplicación de medidas orientadas a su resocialización, esto es, a los condenados por delitos leves y menos graves ${ }^{14}$, generalmente no reincidentes ${ }^{15}$.

14 En una muestra de 285 expedientes de expulsión, Martín Escribano (2015, p. 154) encontró que la pena media de prisión sustituida tenía una duración de 15 meses.

15 Cuando los extranjeros son reincidentes el juez tiende a pensar que deben cumplir la pena de prisión (Roig Torres, 2014; Larrauri, 2016, p. 20). La Circular 7/2015 de la Fiscalía General del Estado asume esta tendencia, al señalar que "la expulsión judicial será, en principio, asumible, salvo circunstancias especiales, cuando la pena de prisión impuesta sea susceptible virtualmente de beneficiarse 
Y aunque se podría pensar que el retorno a su país de residencia favorece su reinserción social, lo cierto es que ese retorno se produce sin control, tratamiento ni asistencia de ningún tipo, sin tener en cuenta dónde tiene el condenado los lazos familiares más fuertes o las mejores perspectivas de trabajo, y en muchas ocasiones contra su voluntad. No es admisible que se argumente que la expulsión penal responde a fines de prevención general, pues no se entiende cómo pueden alcanzarse renunciando a la ejecución de todo o parte de la pena (Rodríguez Yagüe, 2012, pp. 24-25). En segundo lugar, la posibilidad de la expulsión penal o administrativa supone que el reo reciba un diferente trato dentro de la prisión mientras está a la espera de que se ejecute ${ }^{16}$, con consecuencias negativas para sus perspectivas de reinserción. En tercer lugar, aunque tanto la expulsión penal como la administrativa por antecedentes penales recogen el arraigo como causa de denegación, lo cierto es que se opera con un cierto automatismo que no favorece precisamente la individualización de la decisión finalmente adoptada ${ }^{17}$, lo que lleva a

de la suspensión condicional" (p. 12). Si no se impone la expulsión por la gravedad del hecho o las necesidades de prevención general, procede el cumplimiento de la pena de prisión, salvo "en los casos en que el arraigo personal haya sido el fundamento de la inaplicación de la expulsión sustitutiva” (p. 21).

16 Hasta la entrada en vigor de la Instrucción 3/2019, sobre Normas generales sobre internos extranjeros, la Instrucción 21/2011 de la Dirección General de Coordinación Territorial y Medio Abierto de la Secretaría General de Instituciones Penitenciarias, de actualización de la Instrucción 18/2005 sobre Normas Generales sobre Internos Extranjeros, ordenaba diferenciar dos grupos de internos extranjeros: los que tenían la documentación en orden, y en principio podrían quedarse en España, que podían seguir la vía de la reinserción y beneficiarse de los permisos y eventualmente del régimen abierto, y los que no, respecto de los cuales había que alentar el uso de los mecanismos de repatriación. En la práctica, la inclusión en este último grupo solía significar ausencia de permisos y el cumplimiento íntegro de la condena en segundo grado sin libertad condicional (García España, 2007, 2016, pp. 23-24, 2018, pp. 137 138; Rodríguez Yagüe, 2012, pp. 25 ss.; Brandariz García, 2014; Ríos Martín et al., 2015; Larrauri, 2016, pp. 21-22).

17 Las reformas que ha experimentado el art. $89 \mathrm{CP}$ se han orientado, precisamente, a lograr que la expulsión constituya la regla, mientras que el cumplimiento efectivo de la pena en España debería ser la excepción (Brandariz García, 2007, p. 120; Salvador Concepción, 2012). Ya en el año 2006 la Fiscalía General del Estado, en su Circular 2/2006 sobre diversos aspectos relativos al régimen de los extranjeros en España (p. 5), reconoció que la aplicación de la medida de expulsión tenía un carácter más imperativo que facultativo. 
expulsar a condenados cuyas mejores perspectivas de resocialización se encuentran precisamente en España.

En conjunto, cabe apuntar que la lógica que informa la expulsión de condenados extranjeros nada tiene que ver con sus posibilidades de reinserción y rehabilitación social, fines a los que se orientan tanto la ejecución de la pena de prisión como el reconocimiento mutuo de condenas a penas de prisión y de resoluciones de libertad vigilada. Los instrumentos españoles dirigidos a la expulsión están interfiriendo con los comunitarios orientados al reconocimiento mutuo, obstaculizando que estos últimos sean efectivos, lo que a medio plazo dificulta la cooperación judicial europea y la consecución de un verdadero espacio europeo de libertad, seguridad y justicia. Al promocionar la expulsión de condenados comunitarios, en lugar del reconocimiento mutuo de sus sentencias, no se tiene en cuenta que el objetivo de vaciar las cárceles de extranjeros, protegiendo al mismo tiempo a las víctimas y al público en general, se puede conseguir de manera más permanente y efectiva a través de la rehabilitación y reinserción social, pues no son objetivos contradictorios, sino complementarios.

\section{REFERENCIAS}

Acale Sánchez, M. (2010). Libertad vigilada. En F. J. Álvarez García y J. L. González Cussac (Dirs.), Comentarios a la Reforma Penal de 2010 (pp. 147-158). Valencia: Tirant lo Blanch.

Aebi, M. (2016). Inmigración y delincuencia. En Aebi, M. (ed.), Aspectos esenciales de la Criminología actual (pp. 109-174). Barcelona: UOC.

Aranda Carbonel, M. J. (2007). Reeducación y reinserción social. Madrid: Ministerio del Interior.

Banks, J. (2011) Foreign national prisoners in the UK: explanations and implications. The Howard Journal of Criminal Justice, 50(2), 184-198.

Bhui, H. S. (2009). Foreign National Prisoners: Issues and Debates. En H. S. Bhui (Ed.). Race and Criminal Justice (pp. 154-169). London: Sage.

Blanco Cordero, I. y Doval Pais, A. (2013). La extranjería como factor de discriminación de los condenados a penas de prisión. En L. R. Ruiz Rodríguez (Ed.), Crimen organizado y extranjería en España y Marruecos (pp. 51-84). Valencia: Tirant lo Blanch.

Boza Martínez, D. (2016). La expulsión de personas extranjeras condenadas penalmente. Cizur Menor: Thomson Reuters Aranzadi.

Brandariz García, J. A. (2010). Resocialización e inclusión en el tratamiento punitivo de los migrantes. En M. J. Bernuz Beneitez y R. Susín Betrán 
(Coords.), Seguridad, excepción y nuevas realidades jurídicas (pp. 103132). Granada: Comares.

Brandariz García, J. A. (2011). Sistema penal y control de los migrantes. Gramática del migrante como infractor penal. Granada: Comares.

Brandariz García, J. A. (2014). El gobierno de la penalidad. Madrid: Dykinson.

Brandariz García, J. A. (2016). Crimmigration policies and the Great Recession: Analysis of the Spanish case. En M. J. Guia, R. Koulish y V. Mitsilegas (Eds.), Immigration Detention, Risk and Human Rights (pp. 185-197). New York: Springer.

Brandariz García, J. A. y Fernández Bessa, C. (2017). La crimigración en el contexto español: el creciente protagonismo de lo punitivo en el control migratorio. En A. López-Sala y D. Godenau (Coords.), Estados de contención, estados de detención. El control de la inmigración irregular en España (pp. 119-143). Barcelona: Anthropos.

Cid Moliné, J. (2010). La política criminal europea en material de sanciones alternativas a la prisión y la realidad española: una brecha que debe superarse. En C. Arangüena Fanego (Dir.), Espacio europeo de libertad, seguridad y justicia: últimos avances en cooperación judicial penal (pp. 268-289). Valladolid: Lex Nova.

Cugat Mauri, M. (2001). La expulsión de extranjeros: política migratoria y funciones del Derecho penal. Revista de Derecho y Proceso Penal, 6, 23-37.

Daunis Rodríguez, A. (2009). El derecho penal como herramienta de la política migratoria. Granada: Comares.

De Bondt, W. y Suominen, A. (2015). State Responsibility When Transferring Non-consenting Prisoners to Further their Social Rehabilitation Lessons Learnt from the Asylum Case Law. European Criminal Law Review, 5(3), 347-370.

De Marcos Madruga, F. (2010). Una aproximación al tratamiento penitenciario de los extranjeros en prisión. Diario La Ley, 7410, 26 de mayo de 2010.

De Wree, E., Vander Beken, T. y Vermeulen, G. (2009). The transfer of sentenced persons in Europe. Punishment and Society, 11(1), 111-128.

Defensor del Pueblo (2018). Mecanismo Nacional de Prevención de la Tortura. Informe anual 2017. Recuperado de https://www.defensordelpueblo. es/informe-mnp/mecanismo-nacional-prevencion-la-tortura-informeanual-2017/

Defensor del Pueblo (2019). Mecanismo Nacional de Prevención de la Tortura. Informe anual 2018. Recuperado de https://www.defensordelpueblo. es/informe-mnp/mecanismo-nacional-prevencion-la-tortura-informeanual-2018/

Delgrande, N. y Aebi M. F. (2010). Las estadísticas sobre los reclusos extranjeros en Europa (1989-2006). En S. Palidda S. y J. A. BrandarizGarcía (Eds.), Criminalización racista de los migrantes en Europa (pp. 135-150). Granada: Comares. 
Durnescu, I. (2017). Framework decisions 2008/947 and 2009/829: state of play and challenges. ERA Forum, 18(1), 355-363.

European Union Agency on Fundamental Rights (2016). Criminal detention and alternatives: fundamental rights in EU cross-border transfers. Luxembourg: Publications Office of the European Union. Recuperado de http://fra.europa.eu/en/publication/2016/criminal-detention-andalternatives-fundamental-rights-aspects-eu-cross-border

Fernández Arévalo, L. (2010). Expulsión judicial y reforma de la LO 5/2010 (I). Revista de Derecho Migratorio y Extranjería, 24, 11-36.

Fernández Bessa, C. (2013). Il panorama dei Centri di internamento per stranieri in Spagna: Dal controllo delle frontiere alla gestione della criminalità. Antigone, 8(1), 68-91.

Fernández Bessa, C. y Brandariz García, J. A. (2016). Transformaciones de la penalidad migratoria en el contexto de la crisis económica: El giro gerencial del dispositivo de deportación. InDret: Revista para el Análisis del Derecho, 4, 1-25. Recuperado de https://www.raco.cat/index.php/ InDret/article/view/314500/404653

Fiscalía General del Estado (2014). Memoria elevada al Gobierno de S.M. presentada al inicio del año judicial por el Fiscal General del Estado Excmo. Sr. D. Eduardo Torres-Dulce Lifante. Volumen I. Madrid: Ministerio de Justicia. Recuperado de https:/www.fiscal.es/fiscal/PA_WebApp_SGNTJ_NFIS/ descarga/MEMFIS14.pdf?idFile=dd3ff8fc-d0c5-472e-84d2-231be24bc4b2

Flore, D., Bosly, S., Honhon, A. y Maggio, J. (Eds.) (2012). Probation measures and alternative sanctions in the European Union. Cambridge: Intersentia.

García España, E. (2007). Extranjeros presos y reinserción: Un reto del siglo XXI. En A. I. Cerezo Domínguez y E. García España (Eds.), La prisión en España: Una perspectiva criminológica (pp. 101-134). Granada: Comares.

García España, E. (2016). La expulsión como sustitutivo de la pena de prisión en el Código Penal de 2015. ¿De la discriminación a la reinserción? Revista Electrónica de Ciencia Penal y Criminología, 18-07, 1-31.

García España, E., Becerra Muñoz, J. y Aguilar Conde, A. (2012). Realidad y política penitenciarias. Málaga: Instituto Andaluz Interuniversitario de Criminología.

González Sánchez, I. (2015). Encarcelamiento y política neoliberal: Incremento de presos y funciones de la prisión. En D. Ávila y S. García (eds.). Enclaves de riesgo. Gobierno neoliberal, desigualdad y control social (pp. 267-278). Madrid: Traficantes de Sueños.

González Sánchez, I. (2016). La penalización de los migrantes: irregularidad y cárcel en la construcción del Estado neoliberal. Migraciones, 39, 123-147. Recuperado de http://revistas.upcomillas.es/index.php/ revistamigraciones/article/view/6833/6644

Guisasola Lerma, C. (2010). La reforma del Código penal en materia de expulsión judicial de extranjeros como medida sustitutiva de penas privativas de libertad (art. 89). En F. J. Álvarez García y J. L. González 
¿Fracaso del reconocimiento mutuo de resoluciones de libertad vigilada en España? 173

Cussac (Dirs.), Comentarios a la Reforma Penal de 2010 (pp. 131-137). Valencia: Tirant lo Blanch.

Kaufman, E. (2012). Finding foreigners: race and the politics of memory in British prisons. Population, Space and Place, 18(6), 701-714.

Knapen, M. (2010). Implementation of Framework Decisions on the Enforcement of Foreign Criminal Judgments: (How) Can the Aim of Resocialisation be Achieved? En M. Groenhuijsen, T. Kooijmans y T. de Roos (Eds.), Fervet Opus. Liber Amicorum Anton van Kalmthout (pp. 113123). Apeldoordn-Antwerpen-Portland: Maklu.

Larrauri Pijoan, E. (2016). Antecedentes penales y expulsión de personas inmigrantes. InDret: Revista para el Análisis del Derecho, 2, 1-29. Recuperado de http://www.indret.com/pdf/1214.pdf

Leganés Gómez, S. (2002). Clasificación penitenciaria, permisos de salida y extranjeros en prisión. Madrid: Dykinson.

Leganés Gómez, S. (2016). Orden europea relativa a medidas de sustitución de prisión provisional y libertad vigilada del agresor. Diario La Ley, 8704, 17 de febrero de 2016.

Martín Escribano, P. A. (2015). La expulsión de extranjeros del artículo 89 del Código Penal. Análisis jurídico penal y criminológico. Tesis doctoral inédita. Recuperado de https://www.tdx.cat/handle/10803/361400

Martínez Escamilla, M. (2009). Inmigración, Derechos Humanos y Política Criminal: ¿Hasta dónde estamos dispuestos a llegar? InDret, 3, 1-44. Recuperado de http://www.indret.com/pdf/651.pdf

Martufi, A. (2018). Assessing the resilience of 'social rehabilitation' as a rationale for transfer: A commentary on the aims of Framework Decision 2008/909/JHA. New Journal of European Criminal Law, 9(1), 43-61.

McNally, G. y Burke, I. (2012). Implementation of the Framework Decision on the transfer of Probation Measures between States in the European Union. EuroVista, 2(2), 70-77.

Ministerio del Interior (2019). Anuario estadístico del Ministerio del Interior 2018. Madrid: Ministerio del Interior. Recuperado de http://www.interior. gob.es/documents/642317/1204854/Anuario+Estad\%C3\%ADstico+del+ Ministerio+del+Interior+2018/5a35fad7-5386-44fb-83ae-9b14e678cc4a

Mitsilegas, V. (2016). EU after Lisbon. Rights, Trust and the Transformation of Justice in Europe. Oxford and Portland, Oregon: Hart.

Monclús Masó, M. (2006). La discriminación de los inmigrantes en el sistema penal español. En R. Bergalli (Coord.), Flujos migratorios y su (des)control. Puntos de vista pluridisciplinarios (pp. 159-200). Barcelona, Anthropos.

Monclús Masó, M. (2008). La gestión penal de la inmigración. El recurso al sistema penal para el control de los flujos migratorios. Buenos Aires: Editores del Puerto.

Montero Pérez de Tudela, E. (2015). Las medidas repatriativas en el ámbito penitenciario: especial mención al traslado de personas condenadas a la luz de las nuevas reformas legislativas. La Ley Penal, 115, 1-23. 
Morgenstern, C. (2009). European Initiatives for Harmonisation and Minimum Standards in the Field of Community Sanctions and Measures. European Journal of Probation, 1(2), 128-141.

Neveu, S. (2016). Le transfert de l'exécution des peines privatives et restrictives de liberté en droit européen. À la recherche d'un équilibre entre intérêts individuels et collectifs. Limal: Anthemis.

Ríos Martín, J. C., Rodríguez Saez, J. A. y Pascual Rodríguez, E. (2015). Manual jurídico para evitar el ingreso en la cárcel. Granada: Comares.

Rodríguez-Medel Nieto, C. y Sebastián Montesinos, A. (2015). Manual práctico de reconocimiento mutuo penal en la Unión Europea. Valencia: Tirant lo Blanch.

Rodríguez Yagüe, C. (2004). Los derechos de los extranjeros en las prisiones españolas. Universidad de Castilla La Mancha.

Rodríguez Yagüe, C. (2012). El modelo político-criminal español frente a la delincuencia de inmigrantes. Revista Electrónica de Ciencia Penal y Criminología, 14-07, 1-42. Recuperado de http://criminet.ugr.es/recpc/14/ recpc14-07.pdf

Roig Torres, M. (2014). La expulsión de los extranjeros en el proyecto de reforma del Código penal. Análisis desde la perspectiva del TEDH. Unas notas sobre el Derecho británico. Estudios Penales y Criminológicos, XXXIV, 423-509. Recuperado de http://www.usc.es/revistas/index.php/ epc/article/view/2114

Ruiz Rodríguez, L. R. (2006). Extranjeros en prisión: una marginación reiterada. En L. R. Ruiz Rodríguez (Dir.). Sistema penal y exclusión de extranjeros (pp. 181-193). Albacete: Bomarzo.

Ruiz Rodríguez, L. R. (2009) Extranjeros en prisión en España. En L. R. Ruiz Rodríguez (Dir.). Respuestas internacionales a los retos de seguridad. Valencia: Tirant lo Blanch.

Salvador Concepción, R. (2012). Problemas que presenta la expulsión del extranjero como medida sustitutiva de su condena penal. Revista de Derecho y Proceso Penal, 28, 131-151.

Sánchez Yllera, I. (1990). Extranjeros en prisión: doble condena. Jueces para la democracia, 10, 65-70.

Úbeda de los Cobos, J. J. (2006). La modificación del régimen de expulsión de extranjeros como sustitutivo de la pena de prisión en la reforma del código penal. Diario La Ley, 6577, 1-5.

Ugelvik, T. (2014). The Incarceration of Foreigners in European Prisons. En S. Pickering y J. Ham (eds.), The Routledge Handbook on Crime and International Migration (pp. 107-120). London and New York: Routledge.

Van Kalmthout, A. M. y Durnescu, I. (2008). European Probation Service Systems. A comparative overview. En A. M. van Kalmthout y I. Durnescu (Eds.), Probation in Europe (pp. 1-42). Nijmegen: Wolf.

Van Zyl Smit, D. y Snacken, S. (2009). Principles of European Prison Law and Policy. Penology and Human Rights. Oxford: Oxford University Press. 
Vermeulen, G. (2007). Mutual instrumentalization of criminal and migration law from an EU perspective. European Journal of Migration and Law, 9(3), 347-361.

Vermeulen, G. et al. (2011). Cross-border execution of judgments involving deprivation of liberty in the EU. Overcoming legal and practical problems through flanking measures. Amberes-Apeldoorn-Portland: Maklu. 